\title{
Nonlinear effects on motions and loads using an iterative time-frequency solver
}

\author{
D. Bruzzone, C. Gironi and A. Grasso \\ Department of Naval Architecture, University of Genova, Italy
}

\begin{abstract}
A weakly nonlinear seakeeping methodology for predicting motions and loads is presented in this paper. This methodology assumes linear radiation and diffraction forces, calculated in the frequency domain, and fully nonlinear FroudeKrylov and hydrostatic forces, evaluated in the time domain. The particular approach employed here allows to overcome numerical problems connected to the determination of the impulse response functions. The procedure is divided into three consecutive steps: evaluation of dynamic sinkage and trim in calm water that can significantly influence the final results, a linear seakeeping analysis in the frequency domain and a weakly nonlinear simulation. The first two steps are performed employing a three-dimensional Rankine panel method. Nonlinear Froude-Krylov and hydrostatic forces are computed in the time domain by pressure integration on the actual wetted surface at each time step. Although nonlinear forces are evaluated into the time domain, the equations of motion are solved in the frequency domain iteratively passing from the frequency to the time domain until convergence. The containership S175 is employed as a test case for evaluating the capability of this methodology to correctly predict the nonlinear behavior related to wave induced motions and loads in head seas; numerical results are compared with experimental data provided in literature.
\end{abstract}

KEY WORDS: Non linear ship motions; Blended methods.

\section{INTRODUCTION}

Since long time seakeeping problems have been mostly studied using linear methods based into the frequency domain. However, when considering increasing wave heights, they cannot deal with important non linear effects that influence motions, forces and, especially, wave loads. Different formulations have been proposed in literature in order to include nonlinear effects in the evaluation of motions and loads in waves. They are solved in the time domain both with two and three dimensional approaches. These methods are generally based on potential flow theory and often use simplifying hypotheses to reduce the complexity involved in facing fully nonlinear methods. Recently, a growing number of studies have also been carried out in order to deal with the viscous flow seakeeping problem, solving the Reynold averaged Navier-Stokes equations in the time domain. These methods are very promising but still require considerable computational time and resources. An extensive bibliography can be found in literature, but a comprehensive classification and review is given in Beck and Reed (2000).

Some approaches (sometime called "hybrid" or "blended"

Corresponding author: Dario Bruzzone

e-mail: bruzzone@dinav.unige.it methods) allow considering a few nonlinear effects, generally related to hydrostatic and Froude-Krylov forces, which are in fact easy to compute in time domain in their intrinsic nonlinear form, by pressure integration over the instantaneous wetted surface. Diffraction and radiation forces are instead obtained transforming in the time domain their frequency domain counterparts. These methods, which can be employed in a wide range of applications, have been developed because of the problems associated with fully nonlinear computations (for instance, numerical stability and wave breaking) and in order to reduce computational time and resources required, but have proved to provide satisfactory results for engineering purpose in a wide range of sea states. For the numerical simulations here proposed, a three-dimensional Rankine panel method has been employed for both the steady state and the linear seakeeping problems. Then, in order to take into account nonlinearities, a blended method of the family in the foregoing description has been used in an alternative dual approach: Froude-Krylov and hydrostatic forces are evaluated in the time domain and the equations of motion are solved in the frequency domain (in their weakly nonlinear form) by an iterative procedure. A short presentation of the basic theory and of the numerical method will be given at first, then the application to a case study for which experimental data are available will be described and discussed. 


\section{FORMULATION}

The main steps of the procedure employed are represented by the consecutive solution of three different problems: the steady flow around a ship advancing at constant speed, for determining iteratively the dynamic sinkage and trim; the unsteady hydrodynamic problem in the frequency domain, used to evaluate radiation and diffraction forces; the weakly nonlinear seakeeping analysis. The present approach is based on the assumptions of inviscid fluid and irrotational flow, which allows the employment of a potential theory. The following formulation is referenced to an orthogonal coordinate inertial system $(x, y, z)$ advancing at the vessel speed $U$. The $x y$ plane coincides with the undisturbed free surface, $x$ is the symmetry axis of the still water plane and is assumed positive astern, $z$-axis is positive upwards.

\section{Analysis of the unsteady problems in the frequency domain}

For a proper number of meaningful encounter frequencies $\omega_{e}$ ship motions are defined by the instantaneous position of a body fixed reference system with respect to the previous system and may be described by a vector $n_{k}(t)$, with $\mathrm{k}=1, \ldots, 6$. A regular incident wave $\eta t=\Re a e^{i \omega_{e} t}$ is assumed. The hypothesis of small motion amplitudes allow us to express the motions as:

$\eta_{k} t=\Re \zeta_{k} \omega_{e} e^{i \omega_{e} t}$

where $\xi_{k}\left(\omega_{e}\right)$ is the complex amplitude of the $k^{\text {th }}$ motion component and $\omega_{e}$ the encounter frequency. $\eta_{k}$ can be determined solving the following linear system of complex equations:

$$
\sum_{k=1}^{6}\left[-\omega_{e}^{2} M_{j k}+A_{j k}+i \omega_{e} B_{j k}+C_{j k}\right] \zeta_{k}=F_{j} \omega_{e}
$$

where $j=1,2,3$ refers to the $x, y, z$ force components respectively and $j=4,5,6$ to the corresponding three moment components. $M_{j k}$ and $C_{j k}$ represent the mass and hydrostatic restoring matrix; $A_{j k}$ and $B_{j k}$ are the added-mass and the damping coefficients; finally $F_{j}\left(\omega_{e}\right)$ are the complex amplitudes of the exciting forces and can be expressed as the sum of incident Froude Krilov and diffraction forces respectively:

$$
F_{j}\left(\omega_{e}\right)=F_{j}^{F K}\left(\omega_{e}\right)+F_{j}^{D}\left(\omega_{e}\right)
$$

The determination of the relevant quantities $A_{j k}, B_{j k}$ and $F_{j}^{D}\left(\omega_{e}\right)$ is carried out solving a set of boundary value problems which are posed in terms of a total velocity potential $\Phi$ that must satisfy the Laplace equation $\Phi \Delta=0$ into the fluid domain coupled with nonlinear boundary conditions on the free surface and on the hull surface. Linearization of the relevant boundary conditions is possible recurring to a perturbation approach in which some quantities are assumed of a smaller order of magnitude around some suitable base flow potential. The steady potential, the double model potential or the free stream potential can be assumed at a descending order as the basis flow.

The total potential can be expressed as the sum of the potential of a steady flow $\left(\Phi_{S}\right)$ and of a perturbation unsteady potential ( $\Phi_{U S}$ ); in turn, the unsteady perturbation potential may be written as superposition of an incident wave potential $\left(\Phi_{F K}\right)$, of a diffraction potential $\left(\Phi_{D}\right)$ and of six radiation potentials $\left(\Phi_{k}\right)$.

$\Phi=\Phi_{S}+\Phi_{U S}=\Phi_{S}+\Phi_{F K}+\Phi_{D}+\sum_{k=1}^{6} \Phi_{k}$

By assuming the steady potential as a term of leading order, a first linearization can be carried out for the unsteady problems. The steady potential may be also expressed as the sum of some base flow and of a lower order steady perturbation. Denoting with $\vec{n} \equiv\left(n_{1}, n_{2}, n_{3}\right)$ the unit normal and with $\left(n_{4}, n_{5}, n_{6}\right) \equiv \vec{r} \times \vec{n}$, the hull boundary conditions are:

$$
\left\{\begin{array}{l}
\frac{\partial \Phi_{k}}{\partial n}=i \omega_{e} n_{k}+m_{k} \\
\frac{\partial \Phi_{D}}{\partial n}=-\frac{\partial \Phi_{F K}}{\partial n}
\end{array}\right.
$$

for the six radiation potentials and for the diffraction potential respectively, where the $m_{k}$ are the components of the vector $-\vec{n} \cdot \nabla\left(\nabla \Phi_{S}\right)$ for $k=1,2,3$ and of the vector $-\vec{n} \cdot \nabla\left(\vec{r} \times \nabla \Phi_{S}\right)$ for $k=4,5,6$

On the free surface, the linearized boundary conditions can be expressed via the linear operator $\mathcal{L}$ as:

$$
\left\{\begin{array}{l}
\mathcal{L}\left(\Phi_{k}\right)=\left[-\omega_{e}^{2}+g \frac{\partial}{\partial z}-2 i \omega_{e} \nabla \Phi_{S} \cdot \nabla\right. \\
\left.+\nabla \Phi_{S} \cdot \nabla\left(\nabla \Phi_{S} \cdot \nabla\right)+\frac{1}{2} \nabla\left(\nabla \Phi_{S} \cdot \nabla \Phi_{S}\right) \cdot \nabla\right]\left(\Phi_{k}\right)=0 \\
\mathcal{L}\left(\Phi_{D}\right)=\left[-\omega_{e}^{2}+g \frac{\partial}{\partial z}-2 i \omega_{e} \nabla \Phi_{S} \cdot \nabla\right. \\
\left.+\nabla \Phi_{S} \cdot \nabla\left(\nabla \Phi_{S} \cdot \nabla\right)+\frac{1}{2} \nabla\left(\nabla \Phi_{S} \cdot \nabla \Phi_{S}\right) \cdot \nabla\right]\left(\Phi_{D}\right)=0
\end{array}\right.
$$

To work out all the involved boundary value problems, each of the unknown potentials is expressed in term of a distribution of Rankine sources upon the hull and the free surface: 


$$
\phi(P)=\int_{\partial \Omega} \frac{1}{r(P, Q)} \sigma(Q) d S
$$

The hull and a portion of the free surface are approximated with quadrilateral panels, considering a uniform source strength on each. All the involved boundary value problems are hence solved in terms of these unknown source strengths. A suitable radiation condition is finally posed at the forward border of the computational domain. In the present method radiated and diffracted waves are considered not to propagate ahead the ship and hence it can be applied only for $\omega_{e} U / g=0.25$. A similar approach has been employed for solving the steady flow problem. More details on the methodology can be found in Bruzzone (2003). It should be finally pointed out that, since the free surface computational domain is limited, its extension must be carefully considered in order to avoid wave reflections; moreover, the dimensions of the free surface panels should be chosen taking into account incident, radiated and diffracted wave lengths.

\section{Time domain nonlinear analysis}

Applying the impulse theory (Cummins, 1962), it is possible to write the equations of motion in the time domain as:

$$
\begin{array}{r}
\sum_{k=1}^{6}\left(M_{j k}+A_{j k}^{\infty}\right) \ddot{\eta}_{k}(t)+\int_{0}^{t} h_{j k}(t-\tau) \dot{\eta}_{k}(\tau) d \tau \\
=F_{j}^{D}(t)+F_{j}^{F K}(t)+F_{j}^{H}(t)
\end{array}
$$

with $j=1, \ldots, 6$ and $\dot{\eta}_{k}, \ddot{\eta}_{k}$ the first and the second time derivatives of $\eta_{k} . A^{\infty}$ and $B^{\infty}$ are the infinite-frequency added mass and damping coefficients $F_{j}^{D}(t), F_{j}^{F K}(t)$ and $F_{k}^{H}(t)$ represent the diffraction, Froude-Krylov and hydrostatic forces (and moments) respectively, while $h_{j k}$ are impulse response functions (or retardation functions).

If the hydrostatic forces are considered linearly dependent on the motions and Froude-Krylov and diffraction forces to be linear functions of the wave elevations only, Eq. 8 represents a linear system of differential equations as both coefficients and exciting forces do not depend on motions and on theirs derivatives. If, on the contrary, fully nonlinear hydrostatic and Froude-Krylov forces are introduced, the system results to be nonlinear as the exciting forces depend also on the instantaneous position of the hull and hence on the unknown motions.

The system of Eq. 8, in its nonlinear form, is generally solved in the time domain, evaluating at each time step the accelerations and calculating consequently velocities and displacements by a numerical integration.

As shown by Ogilvie (1964), Eq. 8 and Eq. 2 are related by Fourier transforms and the impulse responses can be derived from the frequency dependent added-mass and damping coefficients and vice versa according to the following relationship:

$$
\begin{array}{r}
h_{j k}=-\frac{2}{\pi} \int_{0}^{\infty} \omega_{e} \pi\left(A_{j k}-A_{j k}^{\infty}\right) \sin \left(\omega_{e} t\right) d \omega_{e} \\
=\frac{2}{\pi} \int_{0}^{\infty}\left(B_{j k}-B_{j k}^{\infty}\right) \cos \left(\omega_{e} t\right) d \omega_{e}
\end{array}
$$

This approach is subjected to some inconveniences and uncertainties, mainly related to the limited range of frequency in which damping and added mass coefficients are known from numerical calculations. Even if the nonlinear forces must be evaluated in the time domain, the system of Eq. 8 can be solved both in the time and in the frequency domain, at least until it is weakly nonlinear. The choice is related to the kind of analysis it is expected to be carried out. For this application the frequency domain has been preferred, as it allows avoiding the initial transient phase and it is faster, as the computational time is connected with the nonlinear degree of the problem and the time step is not constrained by time integration convergence requirements. Denoting with $\mathfrak{I}$ the Fourier transform and with $\mathfrak{I}^{-1}$ its inverse, $\eta_{k}$ can be evaluated by:

$\eta_{k}(t)=\mathfrak{I}^{-1}\left\{\xi_{k}\left(\omega_{e}\right)\right\}$

The Fourier transform into the frequency domain of Eq. 8 can be written as:

$$
\begin{aligned}
\sum_{k=1}^{6} & \left\{-\omega_{e}^{2}\left[M_{j k}+A_{j k}\left(\omega_{e}\right)\right]+i \omega_{e} B_{j k}\left(\omega_{e}\right)\right\} \xi_{k}\left(\omega_{e}\right) \\
& =\mathfrak{J}\left\{F_{j}^{D}(t)\right\}\left(\omega_{e}\right)+\mathfrak{J}\left\{F_{j}^{H}(t)+F_{j}^{F K}(t)\right\}\left(\omega_{e}\right)
\end{aligned}
$$

The system in Eq. 11, in its nonlinear form, cannot be solved directly, because of the nonlinear dependence of Froude-Krylov and hydrostatic forces on ship motions. The following iterative procedure has been hence adopted:

$$
\begin{aligned}
& \sum_{k=1}^{6}\left[-\omega_{e}^{2}\left(M_{j k}+A_{j k}\left(\omega_{e}\right)\right)+i \omega_{e} B_{j k}\left(\omega_{e}\right)+C_{j k}\right] \xi_{k}^{(p)}\left(\omega_{e}\right) \\
& =\mathfrak{I}\left\{F_{j}^{D}(t)\right\}\left(\omega_{e}\right)+\mathfrak{I}\left\{F_{j}^{H}\left(\bar{\eta}^{(p-1)}, t\right)+F_{j}^{F K}\left(\bar{\eta}^{(p-1)}, t\right)\right\}\left(\omega_{e}\right) \\
& +\sum_{k=1}^{6} C_{j k} \xi_{k}^{(p-1)}\left(\omega_{e}\right)
\end{aligned}
$$

where $p$ represents an iteration index and $C_{j k}$ is the linear hydrostatic restoring matrix.

The motions obtained in the previous iteration are transferred in the time domain, where nonlinear FroudeKrylov and hydrostatic forces are evaluated, integrating hydrostatic and hydrodynamic pressure over the actual wetted surface under the incident wave profile. Then the amplitude spectra of the forces are evaluated from the time histories by a Fast Fourier Transform and the equations of 
motion are solved in the frequency domain. The linear solution is used as the first guess.

The $C_{j k}$ terms are considered in both sides of Eq. 12 in addition to the nonlinear hydrostatic forces to render the procedure more robust and as an aid to the convergence of the iterative procedure.

\section{Description of the numerical method}

After the solution of the boundary value problems the relevant forces and coefficients can be determined for calculating the impulse response functions or for the iterative process frequency-time domain.

Different methods in the frequency domain could in principle be chosen to evaluate the quantities necessary for the procedure indicated in the previous paragraph as, for instance, a strip theory, a Rankine source method (Bruzzone and Grasso, 2007) or a 3D theory based on Green functions.

The Rankine source method seems to offer some advantages. It allows performing the three important steps involved in the overall process in a quite homogeneous way. At first it is possible to evaluate the dynamic sinkage and trim in calm water which is the starting mean configuration of the ship. In addition it could be chosen to select the steady potential as the basis potential to solve the various boundary value problems. The method can also deal with multihull marine vehicles and their hydrodynamic interactions. Finally the hull mesh can be used with minor modifications in the complete procedure.

To evaluate the nonlinear Froude-Krylov and hydrostatic forces the hull is described by a structured grid of lines, depending on two normalized parameters $u$ and $v$. At each time step the domain describing the wetted surface is evaluated, as well as the pressure distribution on it. Forces and moment are then calculated by analytical integrations of their distributions treated as bi-cubic function on the domain of the parameter $u$ and $v$.

\section{Loads estimation}

Considering all the forces applied on a given cross section, the dynamic vertical shear force $\left(V_{3}\right)$ can be evaluated as the difference between the vertical component of inertial force $\left(I_{3}\right)$ and the sum of the vertical components of external forces acting on the part of hull forward of the cross section analyzed. As it can be seen in Eq. 13, the external forces here considered are related to radiation $\left(R_{j}\right)$, diffraction $\left(D_{j}\right)$, Froude-Krylov and hydrostatic $\left(F K_{j}\right)$ contributions.

$V_{3}=I_{3}-R_{3}-D_{3}-F K_{3}-H_{3}$

Vertical bending moment $\left(M_{5}\right)$ is calculated in a similar way, considering moments of the forces with respect of the cross section considered:

$M_{5}=I_{5}-R_{5}-D_{5}-F K_{5}-H_{5}$
Assumptions on forces are the same employed for solving the unsteady motion problem, providing a formulation consistent with the previous calculations. Loads due to Froude-Krylov and hydrostatic forces are computed in the time domain considering the inherent non linearity as previously described. Loads due to radiation forces are based on the linear seakeeping calculation even if they depend on nonlinear velocities and accelerations. Inertial loads are due to nonlinear accelerations while loads related to diffraction forces are supposed completely linear and depend only on incident waves.

\section{A CASE STUDY: RESULTS AND DISCUSSION}

In order to test the methodology, the S175 hull has been chosen as an application of the present method. In fact, for this containership experimental data provided by several authors, regarding both motions and loads, are available in literature for different wave amplitudes/length ratios. They can be compared with computed results. In this paper the experimental data provided by Fonseca and Guedes Soares (2004) who analyzed the behavior of a S175 hull model in head regular waves, have been chosen to be correlated. Several wave lengths and three wave slopes have been considered for a Froude number $F_{n}=0.25$.

Fourier analysis of the converged time histories related to each regular wave and to the considered wave slope allows providing the harmonic content. Figs. 1 and 2 represent the non dimensional first harmonic of heave and pitch for three different wave slopes. From these figures, though the nonlinear effects due to the wave slope seem to be adequately captured and manifest the same trend as the experimental results, a sensible overestimation around the peak values of heave and pitch can be noted. This outcome has been thought to be due to a prediction of low values of damping coefficients coming from the frequency domain computations. This behavior appears to be quite common, for this kind of ship, when Rankine sources are employed.

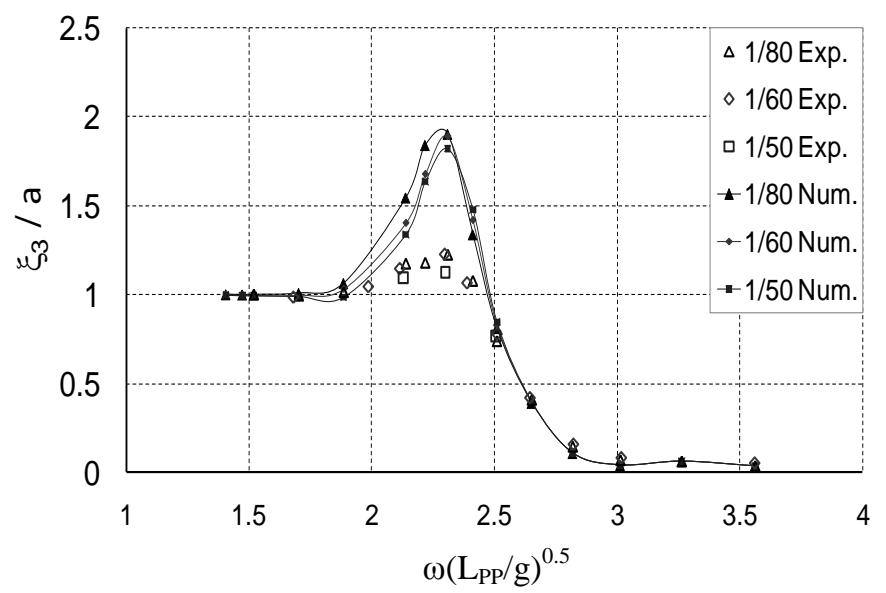

Fig. 1 Heave motion amplitude first harmonic - Amidships. 


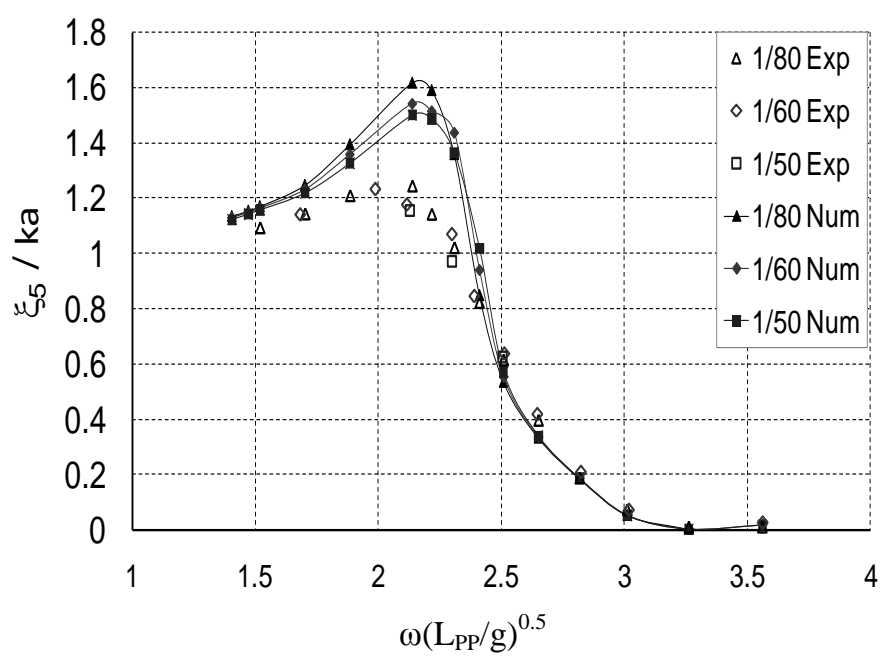

Fig. 2 Pitch motion amplitude first harmonic - Amidships.

Also strip theories tend to overestimate motions at resonance for this vessel, even if in a smaller amount (Pedersen, 2000). On the contrary, as the frequency increases, especially in the pitch motion, after the peak zone, experimental data seem slightly higher than the numerical result, but the extent of this difference at each frequency is anyway small. To overcome the peak overstimation of these damping coefficients could be augmented on the basis of some empirical method. Since in this paper the objective is focused on nonlinear effects, such correction has not been adopted for the results herein presented.

Figs. 3 and 4 present the second harmonics; it should be considered that the order of magnitude of the results is lower than those of the first harmonic, so the differences between experiments and computations are higher in percent. It appears that, as for the case of the first harmonics, the numerical method generally overestimates experimental data. However the overall trend seems to be comparable even if the effect of the wave slope is not always captured.

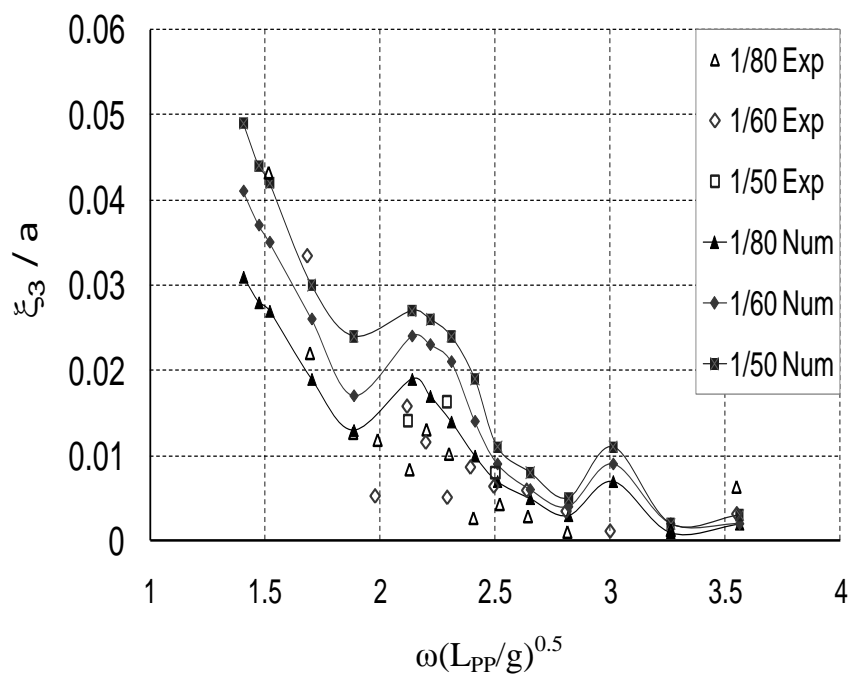

Fig. 3 Heave motion amplitude second harmonic-Amidships.

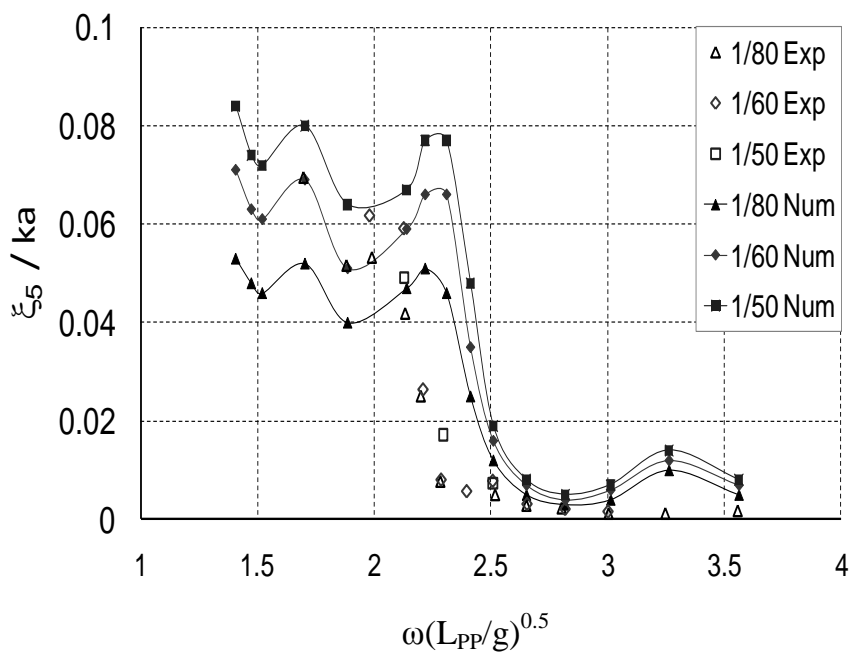

Fig. 4 Pitch motion amplitude second harmonic - Amidships.

A better agreement is observed about the mean values of heave and pitch (i.e. the zero frequency component of the Fourier transform of the time histories) reported in Figs. 5 and 6 . Here, on the basis of the available experimental values, the overall trend and the assessment of the effects of the wave slopes appear to be adequate enough.

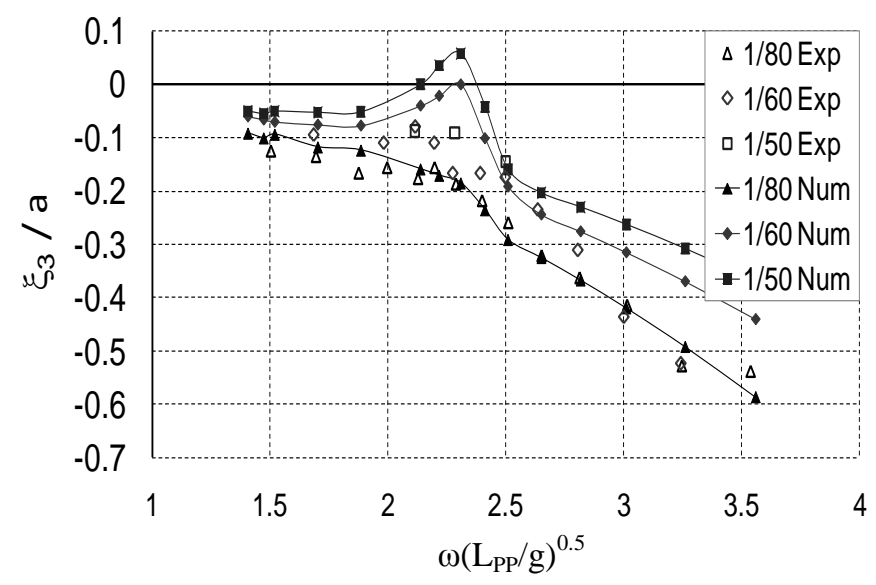

Fig. 5 Heave motion amplitude mean values - Amidships.

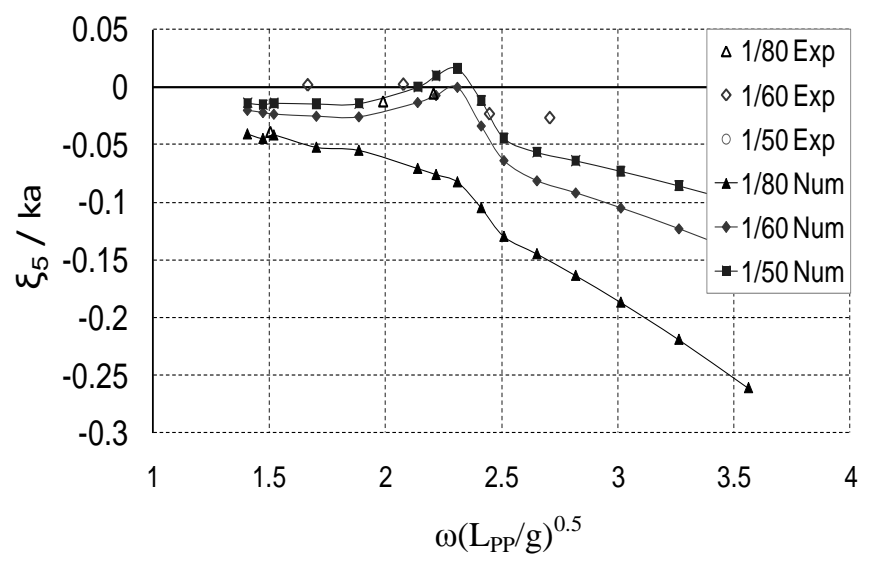

Fig. 6 Pitch motion amplitude mean values - Amidships. 
As regards the wave loads, the first harmonic of vertical shear forces and vertical bending moment at amidships and at station 15 are represented in Figs. 7-10 respectively. The results seem to be in a reasonable agreement with those coming from the experiments, both as general trend and as effects of the wave slopes.

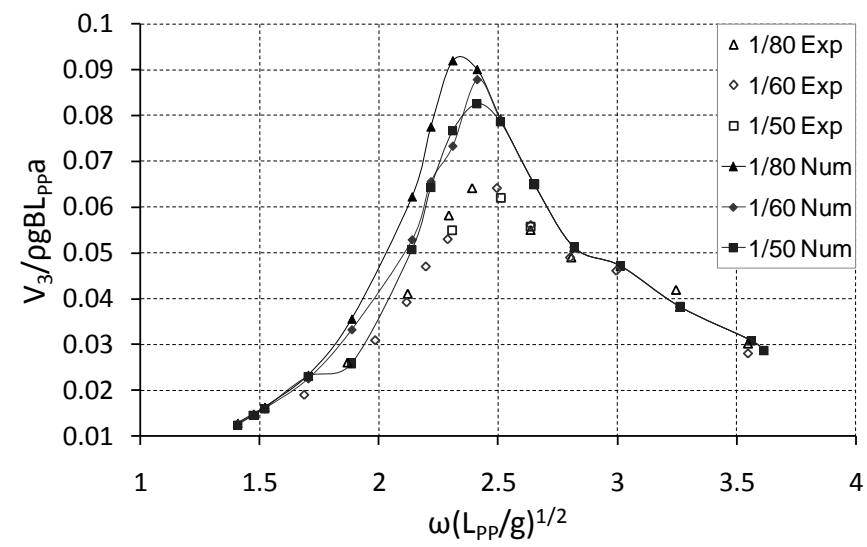

Fig. 7 Vertical shear force amplitude - Amidships.

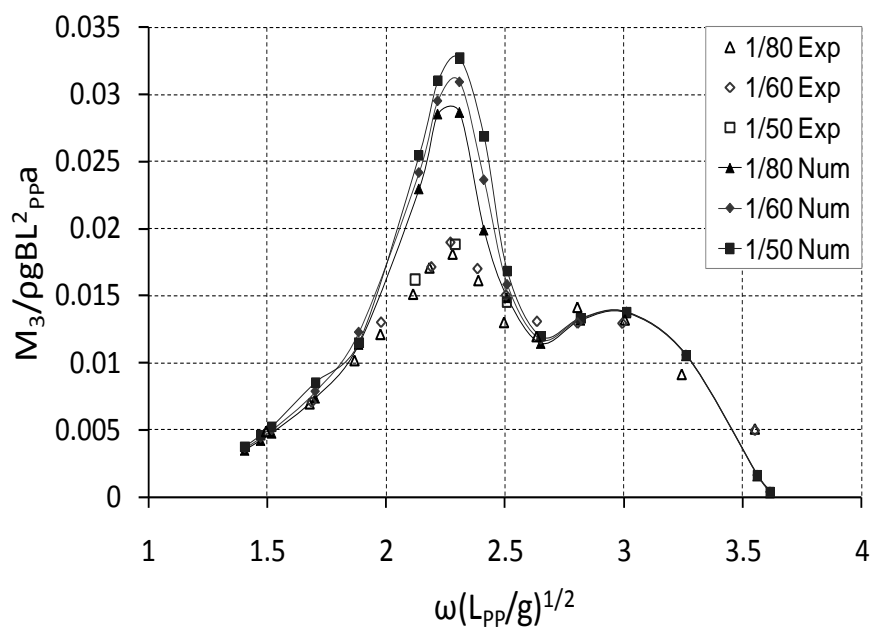

Fig. 8 Vertical bending moment amplitude - Amidships.

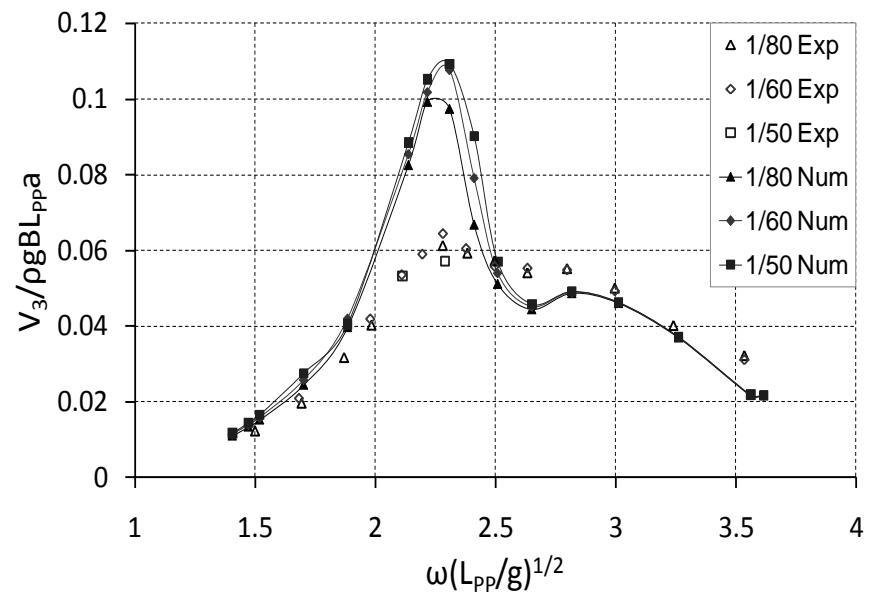

Fig. 9 Vertical shear force amplitude - Station 15.

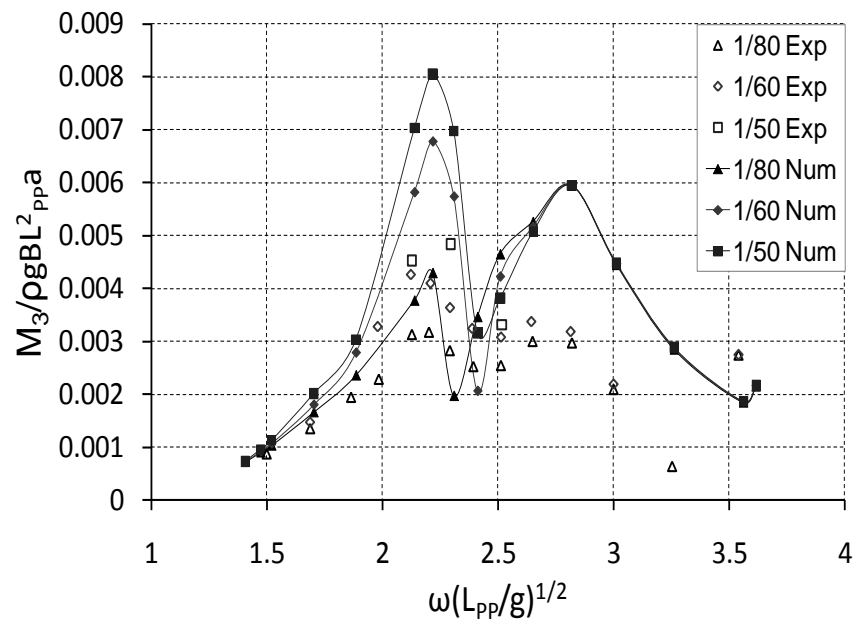

Fig. 10 Vertical bending moment amplitude - Station 15 .

Also in this case numerical values overpredict experimental ones in the frequency ranges of resonance; nevertheless it must be remarked again that the overpredictions in wave motions reflect on loads. The vertical bending moment at station 15 shows a significant overestimation also for higher frequencies. This behavior cannot be related at all to errors in the prediction of motions. Analogous wrong trend was found also by Fonseca and Guedes Soares (2005), who employed a strip theory.

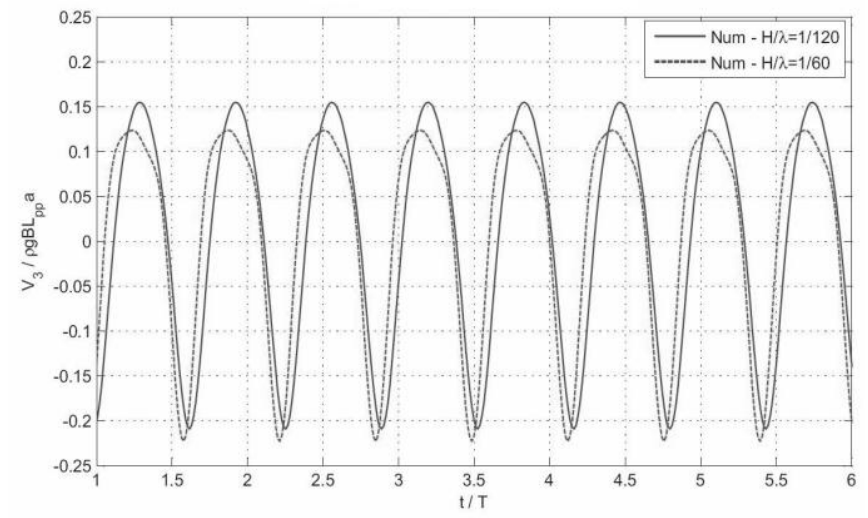

Fig. 11 Time history of vertical shear force at Amidships.

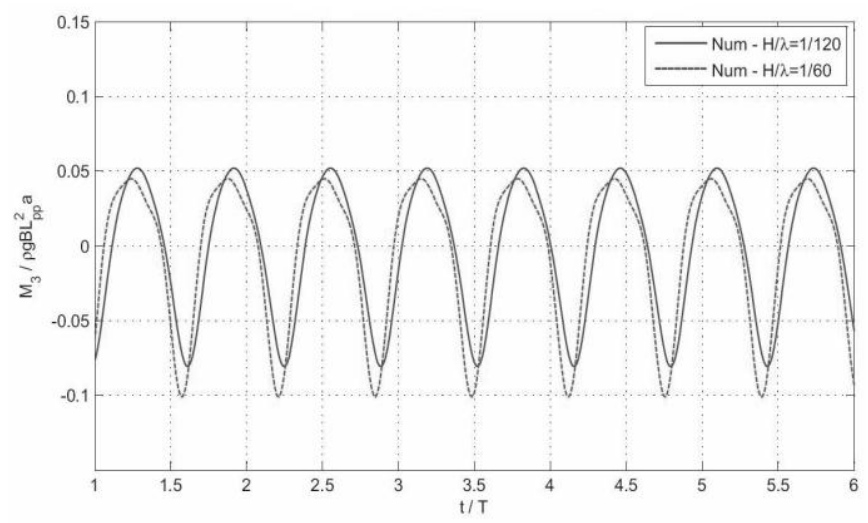

Fig. 12 Time history of vertical bending moment at Amidships. 
Finally Figs. 11-12 represent the time histories of vertical shear and vertical bending moment amidships, where the nonlinear effects causing the differences between hogging and sagging can be clearly noted. It is interesting to highlight how nonlinear effects on loads are found also with small wave amplitudes; this behavior is confirmed by experimental results and can be related to the flare.

\section{CONCLUSIONS}

The paper presents a weakly nonlinear method for evaluating motions and loads in waves. Radiation and diffraction forces are assumed linear whereas Froude Krylov and hydrostatic forces are evaluated in the time domain allowing for their non linearity. With respect to methodologies that adopt similar assumptions, the problem is approached in a different way. A procedure has been proposed that iteratively solves the equation of motions in the frequency domain evaluating only the nonlinear forces in the time domain. This procedure allows reducing computational time and does not require the evaluation of the initial transient phase.

As a test case for this study, the well known S175 containership has been chosen considering its behavior in regular head waves. The obtained numerical results evidence a satisfactory correlation with the experiments, excluding in some cases the resonance frequency range where motions and loads turned out to be overestimated. Trends of non linear effects against wave slopes result adequately predicted even if the accuracy of their evaluation is related to the accuracy in the prediction of the motions. Further studies could be related to the improvement in the prediction of linear forces in order to reduce the overestimation of motions probably due to the lack of damping at resonance.

\section{REFERENCES}

Beck, R. and Reed, A., 2000. Modern seakeeping computations for ships. Twenty-Third Symposium on Naval Hydrodynamics, Val de Reuil, France.

Bruzzone, D., 2003. Application of a Rankine source method to the evaluation of motions of high speed marine vehicles. Proceeding of the 8th International Marine Design Conference, Athens, Greece, Vol. II, pp.69-79.

Bruzzone, D. and Grasso, A., 2007. Nonlinear time domain analysis of vertical ship motions. Archives of Civil and Mechanical Engineering, 7(4), pp. 27-37.

Bruzzone, D. Grasso, A. and Zotti, I., 2008. Nonlinear seakeeping analysis of catamarans with central bulb. Proceedings of the 6th conference on high-performance marine vehicles, Naples, Italy, pp. 47-61.

Cummins, W.E., 1962. The Impulse response function and ship motions. Schiffstechnik, (47), pp. 101-109.

Fonseca, N. and Guedes Soares, C., 2004. Experimental investigation of the nonlinear effects on the vertical motions and loads of a containership in regular waves. Journal of Ship Research, 48 (2), pp. 118-147.

Fonseca, N. and Guedes Soares, C., 2005. Comparison between experimental and numerical results of the nonlinear vertical ship motions and loads on a containership in regular waves. International Shipbuilding Progress, 52(91), pp. 57-89.

Pedersen, T., 2000. Wave Load Prediction - A design tool. Ph.D. Thesis, Technical University of Denmark, Denmark.

Ogilvie, T.F., 1964. Recent progress towards the understanding and prediction of ship motions. Proceedings of the $5^{\text {th }}$ Symposium on Naval Hydrodynamics, 10-12 September Bergen Norway, pp. 380. 